\title{
Noncommutativity of mappings in hybrid fixed point results
}

\author{
Hemant Kumar Pathak and Rosana Rodríguez-López ${ }^{2^{*}}$
}

\author{
"Correspondence: \\ rosana.rodriguez.lopez@usc.es \\ ${ }^{2}$ Departamento de Análisis \\ Matemático, Facultad de \\ Matemáticas, Universidad de \\ Santiago de Compostela, Santiago \\ de Compostela, 15782, Spain \\ Full list of author information is \\ available at the end of the article
}

\begin{abstract}
In this note, some coincidence and common fixed points of nonlinear hybrid mappings have been obtained under certain noncommutativity conditions of mappings. Our results improve several known results in the field of hybrid fixed point theory.

MSC: $54 \mathrm{H} 25 ; 47 \mathrm{H} 10 ; 54 \mathrm{C} 60$

Keywords: coincidence point; fixed point; occasionally coincidentally idempotent; multi-valued mappings
\end{abstract}

\section{Introduction}

As a generalization of the Banach fixed point theorem, Nadler's contraction principle has lead to an excellent fixed point result in the area of nonlinear analysis. Some other works focused on fixed point results for multi-valued mappings are, for instance, [1-5]. Coincidence and common fixed points of nonlinear hybrid contractions (i.e., contractions involving single-valued and multi-valued mappings) have been recently studied by many authors. To mention some of the achievements, we cite, for example, [6-12].

The concept of commutativity of single-valued mappings [13] was extended in [14] to the setting of a single-valued mapping and a multi-valued mapping on a metric space. This concept of commutativity has been further generalized by different authors, viz weakly commuting [15], compatible [16], weakly compatible [8]. It is interesting to note that in all the results obtained so far concerning common fixed points of hybrid mappings the (single-valued and multi-valued) mappings under consideration satisfy either the commutativity condition or one of its generalizations (see, for instance, [6-10]). In this note, we show the existence of fixed points of hybrid contractions which do not satisfy any of the commutativity conditions or its above-mentioned generalizations. Our result extends and improves several well-known results in the field of hybrid fixed point theory. Some other recent related references are $[17,18]$, where common fixed point theorems for hybrid mappings on a symmetric space are proved under the assumptions of weak compatibility and occasional weak compatibility. Some analogous results for the case of contractivity conditions of integral type are presented in [19-21] and generalized contractive hybrid pairs are considered in [22]. Finally, in [23], fixed point results are proved in topological vector space valued cone metric spaces (with nonnormal cones).

(c) 2013 Pathak and Rodríguez-López; licensee Springer. This is an Open Access article distributed under the terms of the Creative Commons Attribution License (http://creativecommons.org/licenses/by/2.0), which permits unrestricted use, distribution, and reproduction in any medium, provided the original work is properly cited. 


\section{Preliminaries}

For a metric space $(X, d)$, let $(C B(X), H)$ and $(C L(X), H)$ denote respectively the hyperspace of non-empty closed bounded and non-empty closed subsets of $X$, where $\mathrm{H}$ is the Hausdorff metric induced by $d$. For $f: X \rightarrow X$ and $T: X \rightarrow C L(X)$, we shall use the following notations:

$$
L(x, y)=\max \left\{d(f x, f y), d(f x, T x), d(f y, T y), \frac{1}{2}(d(f x, T y)+d(f y, T x))\right\}
$$

and

$$
\begin{aligned}
N(x, y)= & {\left[\operatorname { m a x } \left\{d^{2}(f x, f y), d(f x, T x) \cdot d(f y, T y), d(f x, T y) \cdot d(f y, T x),\right.\right.} \\
& \left.\left.\frac{1}{2} d(f x, T x) \cdot d(f y, T x), \frac{1}{2} d(f x, T y) \cdot d(f y, T y)\right\}\right]^{\frac{1}{2}} .
\end{aligned}
$$

We recall some definitions.

Definition 1 Mappings $f$ and $T$ are said to be commuting at a point $x \in X$ if $f T x \subseteq T f x$. The mappings $f$ and $T$ are said to be commuting on $X$ if $f T x \subseteq T f x$ for all $x \in X$.

Definition 2 Mappings $f$ and $T$ are said to be weakly commuting at a point $x \in X$ if

$$
H(f T x, T f x) \leq d(f x, T x) .
$$

The mappings $f$ and $T$ are said to be weakly commuting on $X$ if

$$
H(f T x, T f x) \leq d(f x, T x)
$$

for all $x \in X$.

Definition 3 The mappings $f$ and $T$ are said to be compatible if $f T x \in C B(X)$ for all $x \in X$ and $\lim _{n \rightarrow+\infty} H\left(T f x_{n}, f T x_{n}\right)=0$, whenever $\left\{x_{n}\right\}$ is a sequence in $X$ such that $T x_{n} \rightarrow M \in$ $C B(X)$ and $f x_{n} \rightarrow t \in M$, as $n \rightarrow+\infty$.

Definition 4 The mappings $f$ and $T$ are said to be $f$-weak compatible if $f T x \in C B(X)$ for all $x \in X$ and the following limits exist and satisfy the inequalities:

(i) $\lim _{n \rightarrow \infty} H\left(T f x_{n}, f T x_{n}\right) \leq \lim _{n \rightarrow \infty} H\left(T f x_{n}, T x_{n}\right)$,

(ii) $\lim _{n \rightarrow \infty} d\left(f T x_{n}, f x_{n}\right) \leq \lim _{n \rightarrow \infty} H\left(T f x_{n}, T x_{n}\right)$, whenever $\left\{x_{n}\right\}$ is a sequence in $X$ such that $T x_{n} \rightarrow M \in C B(X)$ and $f x_{n} \rightarrow t \in M$ as $n \rightarrow \infty$.

Let $C(T, f)$ denote the set of all coincidence points of the mappings $f$ and $T$, that is, $C(T, f)=\{u: f u \in T u\}$.

Definition 5 The mappings $f$ and $T$ are said to be coincidentally commuting if they commute at their coincidence points.

Definition 6 Mappings $f$ and $T$ are said to be coincidentally idempotent if $f f u=f u$ for every $u \in C(T, f)$, that is, if $f$ is idempotent at the coincidence points of $f$ and $T$. 
Definition 7 Mappings $f$ and $T$ are said to be occasionally coincidentally idempotent (or, in brief, oci) if ffu $=f u$ for some $u \in C(T, f)$.

It should be remarked that coincidentally idempotent pairs of mappings are occasionally coincidentally idempotent, but the converse is not necessarily true as shown in Example 18 of this note.

\section{Main results}

We recall the following lemma.

Lemma 8 [8] Let $T: Y \rightarrow C B(X)$ and $f: Y \rightarrow X$ be $f$-weak compatible. If $\{f w\}=T w$ for some $w \in Y$ and $H(T x, T y) \leq h(a \cdot L(x, y)+(1-a) \cdot N(x, y))$ for all $x$, $y$ in $Y$, where $0<h<1$, $0 \leq a \leq 1$, then $f T w=T f w$.

We remark that the above-mentioned lemma has been used in $[8,9]$ and [10] to prove the existence of fixed points of hybrid mappings. However, we have noticed some typos in its original statement which have been rectified in the above statement without altering the proof.

Next, we prove a fixed point result for hybrid mappings under a general integral-type contractivity condition. In contrast to [20], we avoid the complete character of the base space $X$, and we introduce hybrid mappings. With respect to the study in [21], we consider here occasionally coincidentally idempotent mappings.

Theorem 9 Let $Y$ be an arbitrary non-empty set, $(X, d)$ be a metric space, $f: Y \rightarrow X$ and $T: Y \rightarrow C B(X)$ be such that

$$
T(Y) \subseteq f(Y)
$$

that is, $\bigcup_{y \in Y} T(y) \subseteq f(Y)$,

there exists $q \in(0,1)$ such that

$$
\int_{0}^{H(T x, T y)} \psi(t) d t \leq q \int_{0}^{L(x, y)} \psi(t) d t \text { for all } x, y \text { in } Y,
$$

$f(Y)$ is complete,

$\psi: \mathbb{R}_{+} \rightarrow \mathbb{R}_{+}$is a Lebesgue measurable mapping which is nonnegative, summable on each compact interval and such that

$$
\psi(x)>0, \quad \forall x>0,
$$

which trivially implies that

$$
\int_{0}^{\epsilon} \psi(t) d t>0 \text { for each } \epsilon>0
$$

and

$$
\int_{0}^{\epsilon} \psi(t) d t<\int_{0}^{\tilde{\epsilon}} \psi(t) d t \quad \text { for each } 0<\epsilon<\tilde{\epsilon} .
$$


Suppose also that

$$
\int_{0}^{\mu \epsilon} \psi(t) d t \leq \gamma(\mu) \int_{0}^{\epsilon} \psi(t) d t \text { for each } \mu>1 \text { and } \epsilon>0,
$$

where $\gamma:(1,+\infty) \longrightarrow \mathbb{R}_{+}$is such that

$$
0<\gamma\left(q^{-1 / 2}\right) \cdot q<1
$$

and

$$
\gamma\left(q^{-1 / 2}\right) \cdot q \cdot \gamma\left(\frac{1}{\gamma\left(q^{-1 / 2}\right) \cdot q}\right) \leq 1
$$

Then $T$ and $f$ have a coincidence point. Further, iff and $T$ are occasionally coincidentally idempotent, then $f$ and $T$ have a common fixed point.

Proof In view of (1) and Nadler's remark in [24], given the point $x_{0} \in Y$, we can construct two sequences $\left\{x_{n}\right\}$ in $Y$ and $\left\{y_{n}\right\}$ in $X$ such that, for each $n \in \mathbb{N}$,

$$
y_{n}=f x_{n} \in T x_{n-1} \quad \text { and } \quad d\left(y_{n}, y_{n+1}\right) \leq q^{-1 / 2} \cdot H\left(T x_{n-1}, T x_{n}\right) .
$$

Indeed, since $T x_{0} \subseteq f(Y)$, there exists $x_{1} \in Y$ such that $f x_{1}=y_{1} \in T x_{0}$. Besides, given $y_{1} \in$ $T x_{0}$, by Nadler's remark in [24] and using that $q^{-1 / 2}>1$, we can choose $y_{2} \in T x_{1} \subseteq f(Y)$ such that $d\left(y_{1}, y_{2}\right) \leq q^{-1 / 2} \cdot H\left(T x_{0}, T x_{1}\right)$ and $y_{2}=f x_{2}$ for a certain $x_{2} \in Y$. The continuation of this process allows to construct the two above-mentioned sequences $\left\{x_{n}\right\}$ and $\left\{y_{n}\right\}$ inductively.

We claim that $\left\{y_{n}\right\}$ is a Cauchy sequence. Using the inequality in (2) and also property (7), which is trivially valid for $\epsilon=0$, it follows, for $n \geq 2$, that

$$
\begin{aligned}
\int_{0}^{d\left(f x_{n-1}, f x_{n}\right)} \psi(t) d t & \leq \int_{0}^{q^{-1 / 2} H\left(T x_{n-2}, T x_{n-1}\right)} \psi(t) d t \\
& \leq \gamma\left(q^{-1 / 2}\right) \int_{0}^{H\left(T x_{n-2}, T x_{n-1}\right)} \psi(t) d t \\
& \leq \gamma\left(q^{-1 / 2}\right) \cdot q \int_{0}^{L\left(x_{n-2}, x_{n-1}\right)} \psi(t) d t
\end{aligned}
$$

where

$$
\begin{aligned}
L\left(x_{n-2}, x_{n-1}\right)= & \max \left\{d\left(f x_{n-2}, f x_{n-1}\right), d\left(f x_{n-2}, T x_{n-2}\right), d\left(f x_{n-1}, T x_{n-1}\right),\right. \\
& \left.\frac{1}{2}\left(d\left(f x_{n-2}, T x_{n-1}\right)+d\left(f x_{n-1}, T x_{n-2}\right)\right)\right\} \\
\leq & \max \left\{d\left(f x_{n-2}, f x_{n-1}\right), d\left(f x_{n-2}, f x_{n-1}\right), d\left(f x_{n-1}, f x_{n}\right), \frac{1}{2} d\left(f x_{n-2}, f x_{n}\right)\right\} \\
\leq & \max \left\{d\left(f x_{n-2}, f x_{n-1}\right), d\left(f x_{n-1}, f x_{n}\right), \frac{1}{2}\left(d\left(f x_{n-2}, f x_{n-1}\right)+d\left(f x_{n-1}, f x_{n}\right)\right)\right\} \\
= & \max \left\{d\left(f x_{n-2}, f x_{n-1}\right), d\left(f x_{n-1}, f x_{n}\right)\right\} .
\end{aligned}
$$


Suppose that

$$
d\left(f x_{n-1}, f x_{n}\right)>\lambda \cdot d\left(f x_{n-2}, f x_{n-1}\right) \quad \text { for some } n \in \mathbb{N} \text { with } n \geq 2 \text {, }
$$

where $\lambda=\gamma\left(q^{-1 / 2}\right) \cdot q \in(0,1)$, hence $d\left(f x_{n-1}, f x_{n}\right)>0$ and

$$
0<\max \left\{d\left(f x_{n-2}, f x_{n-1}\right), d\left(f x_{n-1}, f x_{n}\right)\right\}<\frac{1}{\lambda} d\left(f x_{n-1}, f x_{n}\right),
$$

so that

$$
\begin{aligned}
\int_{0}^{d\left(f x_{n-1}, f x_{n}\right)} \psi(t) d t & \leq \gamma\left(q^{-1 / 2}\right) \cdot q \int_{0}^{\max \left\{d f\left(f x_{n-2}, f x_{n-1}\right), d f\left(x_{n-1}, f x_{n}\right)\right\}} \psi(t) d t \\
& <\gamma\left(q^{-1 / 2}\right) \cdot q \int_{0}^{\frac{1}{\lambda} d\left(f x_{n-1}, f x_{n}\right)} \psi(t) d t \\
& \leq \gamma\left(q^{-1 / 2}\right) \cdot q \cdot \gamma\left(\frac{1}{\lambda}\right) \int_{0}^{d\left(f x_{n-1}, f x_{n}\right)} \psi(t) d t \leq \int_{0}^{d f\left(f x_{n-1}, f x_{n}\right)} \psi(t) d t,
\end{aligned}
$$

where we have also used (6) (a consequence of (4)), (7), (8) and (9). The previous inequalities imply that

$$
\int_{0}^{d\left(f x_{n-1}, f x_{n}\right)} \psi(t) d t<\int_{0}^{d\left(f x_{n-1}, f x_{n}\right)} \psi(t) d t
$$

which is a contradiction. In consequence,

$$
d\left(f x_{n-1}, f x_{n}\right) \leq \lambda \cdot d\left(f x_{n-2}, f x_{n-1}\right), \quad \text { for every } n \in \mathbb{N}, n \geq 2,
$$

where $\lambda=\gamma\left(q^{-1 / 2}\right) \cdot q \in(0,1)$, by hypothesis, and hence $\left\{f x_{n}\right\}$ is a Cauchy sequence in $f(Y)$. This is clear from the following inequality, valid for $n, m \in \mathbb{N}, n>m$,

$$
\begin{aligned}
d\left(f x_{n}, f x_{m}\right) & \leq \sum_{j=m+1}^{n} d\left(f x_{j}, f x_{j-1}\right) \leq \sum_{j=m+1}^{n} \lambda^{j-1} d\left(f x_{1}, f x_{0}\right) \\
& =\frac{\lambda^{m}-\lambda^{n}}{1-\lambda} d\left(f x_{1}, f x_{0}\right) \leq \frac{\lambda^{m}}{1-\lambda} d\left(f x_{1}, f x_{0}\right),
\end{aligned}
$$

which tends to zero as $m \rightarrow+\infty$.

Since $f(Y)$ is complete, then the sequence $\left\{x_{n}\right\}$ has a limit in $f(Y)$, say $u$. Let $w \in f^{-1}(u)$ and prove that $f w \in T w$.

Suppose that $f w \notin T w$, then, by (2), we have

$$
\int_{0}^{d\left(f x_{n+1}, T w\right)} \psi(t) d t \leq \int_{0}^{H\left(T x_{n}, T w\right)} \psi(t) d t \leq q \int_{0}^{L\left(x_{n}, w\right)} \psi(t) d t,
$$

where

$$
\begin{aligned}
L\left(x_{n}, w\right) & =\max \left\{d\left(f x_{n}, f w\right), d\left(f x_{n}, T x_{n}\right), d(f w, T w), \frac{1}{2}\left(d\left(f x_{n}, T w\right)+d\left(f w, T x_{n}\right)\right)\right\} \\
& =d(f w, T w) \quad \text { for } n \text { large. }
\end{aligned}
$$


Here, we have used that $d\left(f x_{n}, f w\right)=d\left(f x_{n}, u\right) \rightarrow 0$, as $n \rightarrow+\infty, d\left(f x_{n}, T x_{n}\right) \leq d\left(f x_{n}, f x_{n+1}\right) \rightarrow$ 0 , as $n \rightarrow+\infty, d(f w, T w)>0$ due to $f w \notin T w$ and $T w$ closed, and

$$
\begin{aligned}
\frac{1}{2}\left(d\left(f x_{n}, T w\right)+d\left(f w, T x_{n}\right)\right) & \leq \frac{1}{2}\left(2 d\left(f x_{n}, f w\right)+d(f w, T w)+d\left(f x_{n}, T x_{n}\right)\right) \\
& \rightarrow \frac{1}{2} d(f w, T w), \quad \text { as } n \rightarrow+\infty .
\end{aligned}
$$

Hence, for $n$ large enough, we have

$$
\int_{0}^{d\left(f x_{n+1}, T w\right)} \psi(t) d t \leq q \int_{0}^{d(f w, T w)} \psi(t) d t
$$

Making $n$ tend to $+\infty$ in the previous inequality, we have

$$
\int_{0}^{d(f w, T w)} \psi(t) d t \leq q \int_{0}^{d(f w, T w)} \psi(t) d t
$$

and, therefore, since $q<1$ and $d(f w, T w)>0$, we get $\int_{0}^{d(f w, T w)} \psi(t) d t<\int_{0}^{d(f w, T w)} \psi(t) d t$, which is a contradiction. Hence $f w \in T w$, that is, $w$ is a coincidence point for $T$ and $f$.

Although this fact is not relevant to the proof, we note that $H\left(T x_{n-1}, T x_{n}\right) \rightarrow 0$ since

$$
\lim _{n \rightarrow \infty} d\left(y_{n-1}, y_{n}\right)=0
$$

Indeed,

$$
\int_{0}^{H\left(T x_{n-1}, T x_{n}\right)} \psi(t) d t \leq q \int_{0}^{L\left(x_{n-1}, x_{n}\right)} \psi(t) d t
$$

where

$$
\begin{aligned}
L\left(x_{n-1}, x_{n}\right) & \leq \max \left\{d\left(f x_{n-1}, f x_{n}\right), d\left(f x_{n}, f x_{n+1}\right)\right\} \\
& \leq \max \left\{d\left(f x_{n-1}, f x_{n}\right), \lambda d\left(f x_{n-1}, f x_{n}\right)\right\}=d\left(f x_{n-1}, f x_{n}\right),
\end{aligned}
$$

therefore

$$
\int_{0}^{H\left(T x_{n-1}, T x_{n}\right)} \psi(t) d t \leq q \int_{0}^{d\left(f x_{n-1}, f x_{n}\right)} \psi(t) d t
$$

Then $\lim _{n \rightarrow+\infty} \int_{0}^{H\left(T x_{n-1}, T x_{n}\right)} \psi(t) d t=0$ and, by the properties of $\psi$, we get $H\left(T x_{n-1}\right.$, $\left.T x_{n}\right) \rightarrow 0$ as $n \rightarrow+\infty$. From the definition of $\left\{y_{n}\right\}$, we deduce that $d\left(f x_{n}, T x_{n}\right) \leq H\left(T x_{n-1}\right.$, $\left.T x_{n}\right)$ for every $n$ and, therefore, $\lim _{n \rightarrow \infty} d\left(f x_{n}, T x_{n}\right)=0$, so that $\left\{x_{n}\right\}$ is asymptotically $T$-regular with respect to $f$. However, this property can be deduced directly from the fact that

$$
0 \leq d\left(f x_{n}, T x_{n}\right) \leq d\left(f x_{n}, f x_{n+1}\right) \rightarrow 0 \quad \text { as } n \rightarrow+\infty
$$


Now, if $f$ and $T$ are occasionally coincidentally idempotent, then $f f w=f w$ for some $w \in$ $C(T, f)$. Then we have

$$
\int_{0}^{H(T f w, T w)} \psi(t) d t \leq q \int_{0}^{L(f w, w)} \psi(t) d t
$$

where

$$
\begin{aligned}
L(f w, w) & =\max \left\{d(f f w, f w), d(f f, T f w), d(f w, T w), \frac{1}{2}(d(f f w, T w)+d(f w, T f w))\right\} \\
& =\max \left\{d(f w, f w), d(f w, T f w), d(f w, T w), \frac{1}{2}(d(f w, T w)+d(f w, T f w))\right\} \\
& =d(f w, T f w) \leq H(T w, T f w) .
\end{aligned}
$$

If $T f w \neq T w$, then from inequality (10) and using (5) (which is guaranteed by (4)), we have that

$$
\int_{0}^{H(T f w, T w)} \psi(t) d t \leq q \int_{0}^{H(T f w, T w)} \psi(t) d t<\int_{0}^{H(T f w, T w)} \psi(t) d t,
$$

which is a contradiction. Hence $T f w=T w$. Thus we have $f w=f f w$ and $f w \in T w=T f w$, i.e., $f w$ is a common fixed point of $f$ and $T$.

Let $\Phi$ denote the family of maps $\phi$ from the set $\mathbb{R}_{+}$of nonnegative real numbers to itself such that

$$
\phi(t) \leq q t \quad \text { for all } t \geq 0 \text { and for some } q \in(0,1) .
$$

Corollary 10 Let $Y$ be an arbitrary non-empty set, $(X, d)$ be a metric space, $f: Y \rightarrow X$ and $T: Y \rightarrow C B(X)$ be such that $T(Y) \subseteq f(Y)$,

$$
\int_{0}^{H(T x, T y)} \psi(t) d t \leq \phi\left(\int_{0}^{L(x, y)} \psi(t) d t\right)
$$

for all $x, y$ in $Y$, where $\phi \in \Phi$ (satisfying (11) for a certain $q \in(0,1))$,

$f(Y)$ is complete,

$\psi: \mathbb{R}_{+} \rightarrow \mathbb{R}_{+}$is a Lebesgue measurable mapping which is nonnegative, summable on each compact interval and such that (4) holds. Suppose also that (7), (8) and (9) hold for a certain $\gamma:(1,+\infty) \longrightarrow \mathbb{R}_{+}$and $q$ determined by (11). Then $T$ and $f$ have a coincidence point. Further, iff and $T$ are occasionally coincidentally idempotent, then $f$ and $T$ have a common fixed point.

Proof It is a consequence of Theorem 9 since (11) and (12) imply that

$$
\int_{0}^{H(T x, T y)} \psi(t) d t \leq \phi\left(\int_{0}^{L(x, y)} \psi(t) d t\right) \leq q \int_{0}^{L(x, y)} \psi(t) d t
$$

for all $x, y$ in $Y$ and $q \in(0,1)$. 
Remark 11 The condition

$$
\int_{0}^{\mu \epsilon} \psi(t) d t \leq \mu \int_{0}^{\epsilon} \psi(t) d t \quad \text { for each } \mu>1 \text { and } \epsilon>0
$$

implies the validity of hypothesis (7) in Theorem 9 for the particular case of $\gamma$ the identity mapping. Moreover, for $0<q<1$, hypotheses (8) and (9) are trivially satisfied for this choice of $\gamma$. Indeed, using that $0<q<1$, we get

$$
0<\gamma\left(q^{-1 / 2}\right) \cdot q=q^{-1 / 2} \cdot q=q^{1 / 2}<1
$$

and

$$
\gamma\left(q^{-1 / 2}\right) \cdot q \cdot \gamma\left(\frac{1}{\gamma\left(q^{-1 / 2}\right) \cdot q}\right)=q^{-1 / 2} q \frac{1}{q^{-1 / 2} q}=1 .
$$

Remark 12 Assuming (8), condition (9) is trivially valid if $\lambda \cdot \gamma\left(\frac{1}{\lambda}\right) \leq 1$ for every $\lambda \in(0,1)$ or, equivalently, $\gamma\left(\frac{1}{\lambda}\right) \leq \frac{1}{\lambda}$ for every $\lambda \in(0,1)$, that is, $\gamma(z) \leq z$ for every $z>1$. Note that this last condition is trivially valid for $\gamma$ the identity mapping. Moreover, if $\gamma(z) \leq z$ for every $z>1$, then $\gamma(z)<z^{2}$ for every $z>1$ and, therefore, if $q \in(0,1)$, then $\gamma\left(q^{-1 / 2}\right)<q^{-1}$, obtaining (8) if $\gamma\left(q^{-1 / 2}\right)>0$.

Remark 13 According to Remark 12 , for $q \in(0,1)$ fixed and $\psi$ satisfying $(4)$, an admissible function $\gamma$ can be obtained by taking

$$
\gamma(z) \geq \sup _{\epsilon>0} \frac{\int_{0}^{z \epsilon} \psi(t) d t}{\int_{0}^{\epsilon} \psi(t) d t}, \quad z>1
$$

provided that $\gamma\left(q^{-1 / 2}\right)>0$ and $\gamma(z) \leq z$ for every $z>1$.

Example 14 Taking $\psi$ as the constant function $\psi(t)=K>0, t>0$, in the statement of Theorem 9, condition (7) is reduced to

$$
K \mu \epsilon \leq \gamma(\mu) K \epsilon \text { for each } \mu>1 \text { and } \epsilon>0 \text {, }
$$

so that we must choose $\gamma$ as a nonnegative function satisfying that $\gamma(z)=z$ for $z>1$ (obviously, $\gamma\left(q^{-1 / 2}\right)>0$ since $\left.q \in(0,1)\right)$ in order to guarantee conditions (7), (8) and (9).

Example 15 A simple calculation provides that, for the function $\psi(t)=t, t>0$, condition (7) is written as $\gamma(z) \geq z^{2}$ for $z>1$ and, therefore, in this case condition (8) is never fulfilled. If we take $\psi(t)=K t^{m}, t>0$, for $K>0$ and $m>0$ fixed, then (7) implies that $\gamma(z) \geq z^{m+1}>z$ for $z>1$.

Example 16 Now, we choose $\psi(t)=K t^{m}, t>0$, where $K>0$ and $-1<m<0$ are fixed. Note that the case $m=0$ has already been studied in Example 14. In this case $-1<m<0$, condition (7) is reduced to

$$
K \frac{(\mu \epsilon)^{m+1}}{m+1} \leq \gamma(\mu) K \frac{\epsilon^{m+1}}{m+1} \text { for } \mu>1 \text { and } \epsilon>0,
$$


which is equivalent to $\gamma(z) \geq z^{m+1}$ for $z>1$. Note that this inequality implies, for $0<q<1$, that $\gamma\left(q^{-1 / 2}\right)>0$. If we add the hypothesis $\gamma(z) \leq z$ for $z>1$, then we guarantee the validity of conditions (8) and (9) due to Remark 12. Hence, we can take any nonnegative function $\gamma$ satisfying that

$$
z^{m+1} \leq \gamma(z) \leq z \quad \text { for } z>1
$$

Of course, $\gamma(z)=z$ and $\gamma(z)=z^{m+1}$ are valid choices.

Example 17 Take $\psi(t)=e^{-t}, t>0$. Condition (7) is equivalent to

$$
1-e^{-\mu \epsilon} \leq \gamma(\mu)\left(1-e^{-\epsilon}\right) \quad \text { for } \mu>1 \text { and } \epsilon>0,
$$

that is,

$$
\gamma(\mu) \geq \frac{1-e^{-\mu \epsilon}}{1-e^{-\epsilon}} \quad \text { for } \mu>1 \text { and } \epsilon>0 .
$$

Now, for each $z>1$ fixed, we calculate $\sup _{\epsilon>0} \frac{1-e^{-z \epsilon}}{1-e^{-\epsilon}}$, which is obviously positive, and we check that its value is equal to $z$.

It is easy to prove that for $z>1$ fixed, the function $\epsilon \in(0,+\infty) \longrightarrow \mathcal{R}_{z}(\epsilon)=\frac{1-e^{-z \epsilon}}{1-e^{-\epsilon}}$ is decreasing on $(0,+\infty)$. Indeed, the sign of its derivative coincides with the sign of the function $v(\epsilon)=z e^{-z \epsilon}\left(1-e^{-\epsilon}\right)-\left(1-e^{-z \epsilon}\right) e^{-\epsilon}$ and also with the sign of $\tau(\epsilon)=z e^{-z \epsilon}\left(e^{\epsilon}-1\right)+$ $e^{-z \epsilon}-1$ for $\epsilon \in(0,+\infty)$. Now, the function $\tau$ is strictly negative on $(0,+\infty)$ since $\tau(0)=$ $\tau\left(0^{+}\right)=0$ and $\tau^{\prime}(\epsilon)=z(1-z) e^{-z \epsilon}\left(e^{\epsilon}-1\right)<0$ for $\epsilon>0$.

Moreover, $\lim _{\epsilon \rightarrow 0^{+}} \mathcal{R}_{z}(\epsilon)=z$ for each $z>1$; in consequence, $\sup _{\epsilon>0} \mathcal{R}_{z}(\epsilon)=z$ for every $z>1$. Therefore, if $\gamma(z) \geq z$ for every $z>1$, then (7) follows. Note also that if $q \in(0,1)$, then $\gamma\left(q^{-1 / 2}\right)>0$. Finally, for $q \in(0,1)$, if we take $\gamma:(1,+\infty) \longrightarrow \mathbb{R}_{+}$such that $\gamma(z)=z$ for $z>1$, we deduce the validity of (7), (8) and (9).

The following example shows that Theorem 9 is a proper generalization of the fixed point results in [7-10].

Example 18 Let $X=\mathbb{R}_{+}$be endowed with the Euclidean metric, let $f: X \rightarrow X$ and $T$ : $X \rightarrow C B(X)$ be defined by $f x=4\left(x^{2}+x\right)$ and $T x=\left[0, x^{2}+7\right]$. Let $\phi: \mathbb{R}_{+} \rightarrow \mathbb{R}_{+}$be defined by $\phi(t)=\frac{1}{4} t$ for all $t \in \mathbb{R}_{+}$. Then mappings $f$ and $T$ are not commuting and also do not satisfy any of its generalizations, viz weakly commuting, compatibility, weak compatibility. Also the mappings $f$ and $T$ are not coincidentally commuting. Note that $f 1 \in T 1$, but $f f 1 \neq f 1$ and so $f$ and $T$ are not coincidentally idempotent, but $f 0 \in T 0$ and $f f 0=f 0$ thus $f$ and $T$ are occasionally coincidentally idempotent. For all $x$ and $y$ in $X$, we have

$$
\begin{aligned}
\int_{0}^{H(T x, T y)} \psi(t) d t & =\int_{0}^{\left|x^{2}-y^{2}\right|} \psi(t) d t=\int_{0}^{\left(\frac{x+y}{4}\right) \cdot \frac{1}{(x+y+1)} \cdot(4|x-y| \cdot(x+y+1))} \psi(t) d t \\
& =\int_{0}^{\left(\frac{x+y}{4}\right) \cdot \frac{1}{(x+y+1)} \cdot\left(4\left|x^{2}-y^{2}+x-y\right|\right)} \psi(t) d t \leq \int_{0}^{\frac{1}{4} d(f x, f y)} \psi(t) d t \\
& \leq \frac{1}{4} \int_{0}^{d(f x, f y)} \psi(t) d t \leq \frac{1}{4} \int_{0}^{L(x, y)} \psi(t) d t=\phi\left(\int_{0}^{L(x, y)} \psi(t) d t\right) .
\end{aligned}
$$


Note that these inequalities are valid if

$$
\int_{0}^{\frac{1}{4} d\left(f x_{x} f y\right)} \psi(t) d t \leq \frac{1}{4} \int_{0}^{d\left(f x_{0} f y\right)} \psi(t) d t
$$

which is satisfied taking, for instance, the constant function $\psi \equiv 1$. On the other hand, $\gamma$ is chosen as the identity map and it satisfies (8) and (9).

Note that 0 is a common fixed point of $f$ and $T$. We remark that the results of [7-9] and [10] cannot be applied to these mappings $f$ and $T$.

Theorem 19 In Theorem 9, we can assume, instead of condition (2), one of the inequalities

$$
\int_{0}^{H(T x, T y)} \psi(t) d t \leq q\left(a \int_{0}^{L(x, y)} \psi(t) d t+b \int_{0}^{N(x, y)} \psi(t) d t\right) \quad \text { for all } x, y \text { in } Y,
$$

or

$$
\int_{0}^{H(T x, T y)} \psi(t) d t \leq q \int_{0}^{a L(x, y)+b N(x, y)} \psi(t) d t \text { for all } x, y \text { in } Y,
$$

where $a, b \geq 0, a+b \leq 1$ and $q \in(0,1)$.

Similarly, in Corollary 10, we can consider one of the contractivity conditions

$$
\int_{0}^{H(T x, T y)} \psi(t) d t \leq \phi\left(a \int_{0}^{L(x, y)} \psi(t) d t+b \int_{0}^{N(x, y)} \psi(t) d t\right) \text { for all } x, y \text { in } Y,
$$

or

$$
\int_{0}^{H(T x, T y)} \psi(t) d t \leq \phi\left(\int_{0}^{a L(x, y)+b N(x, y)} \psi(t) d t\right) \text { for all } x, y \text { in } Y
$$

where $a, b \geq 0, a+b \leq 1$ and $\phi \in \Phi$ (satisfying (11) for a certain $q \in(0,1))$ and the conclusion follows.

Proof It follows from the inequality

$$
N(x, y) \leq L(x, y) \quad \text { for every } x, y
$$

and the nonnegative character of $a, b$ and $\psi$. Indeed, $d^{2}(f x, f y) \leq[L(x, y)]^{2}$,

$$
\begin{aligned}
& d(f x, T x) \cdot d(f y, T y) \leq[L(x, y)]^{2}, \\
& d(f x, T y) \cdot d(f y, T x) \leq \frac{1}{4}(d(f x, T y)+d(f y, T x))^{2} \leq[L(x, y)]^{2}, \\
& \frac{1}{2} d(f x, T x) \cdot d(f y, T x) \leq\left[\max \left\{d(f x, T x), \frac{1}{2}(d(f x, T y)+d(f y, T x))\right\}\right]^{2} \leq[L(x, y)]^{2}, \\
& \frac{1}{2} d(f x, T y) \cdot d(f y, T y) \leq\left[\max \left\{d(f y, T y), \frac{1}{2}(d(f x, T y)+d(f y, T x))\right\}\right]^{2} \leq[L(x, y)]^{2},
\end{aligned}
$$


hence, for instance,

$$
a \int_{0}^{L(x, y)} \psi(t) d t+b \int_{0}^{N(x, y)} \psi(t) d t \leq(a+b) \int_{0}^{L(x, y)} \psi(t) d t \leq \int_{0}^{L(x, y)} \psi(t) d t .
$$

Note that, in cases (16) and (17), it is not necessary to assume the nondecreasing character of the function $\phi$ since, using that $\phi \in \Phi$, we deduce (14) and (15), respectively.

Of course, the function $\phi \equiv 0$ is admissible in the results of this paper.

Note that, taking $a=1$ and $b=0$ in the inequalities of Theorem 19, we obtain the corresponding contractivity conditions of Theorem 9 and Corollary 10. On the other hand, taking $a=0$ and $b=1$ in Theorem 19, we have the following results, which are also corollaries of Theorem 9 .

Corollary 20 Let $Y$ be an arbitrary non-empty set, $(X, d)$ be a metric space, $f: Y \rightarrow X$ and $T: Y \rightarrow C B(X)$ be such that conditions (1), (3) hold and

$$
\int_{0}^{H(T x, T y)} \psi(t) d t \leq q \int_{0}^{N(x, y)} \psi(t) d t \quad \text { for all } x, y \in Y,
$$

where $0<q<1$ and $\psi: \mathbb{R}_{+} \rightarrow \mathbb{R}_{+}$is a Lebesgue measurable mapping which is nonnegative, summable on each compact interval and such that (4) holds. Assume also that (7), (8) and (9) are fulfilled for a certain $\gamma:(1,+\infty) \longrightarrow \mathbb{R}_{+}$. Then $f$ and $T$ have a coincidence point. Further, if $f$ and $T$ are occasionally coincidentally idempotent, then $f$ and $T$ have a common fixed point.

Corollary 21 Let $Y$ be an arbitrary non-empty set, $(X, d)$ be a metric space, $f: Y \rightarrow X$ and $T: Y \rightarrow C B(X)$ be such that conditions (1), (3) hold and

$$
\int_{0}^{H(T x, T y)} \psi(t) d t \leq \phi\left(\int_{0}^{N(x, y)} \psi(t) d t\right) \text { for all } x, y \in Y,
$$

where $\phi \in \Phi$ (satisfying (11) for $q \in(0,1))$ and $\psi: \mathbb{R}_{+} \rightarrow \mathbb{R}_{+}$is a Lebesgue measurable mapping which is nonnegative, summable on each compact interval and such that (4) holds. Assume also that (7), (8) and (9) are fulfilled for a certain $\gamma:(1,+\infty) \longrightarrow \mathbb{R}_{+}$. Then $f$ and $T$ have a coincidence point. Further, if $f$ and $T$ are occasionally coincidentally idempotent, then $f$ and $T$ have a common fixed point.

Let $\eta:[0, \infty) \rightarrow[0,1)$ be a function having the following property (see, for instance, $[6,25])$

$(\mathcal{P})$ For $t \geq 0$, there exist $\delta(t)>0, s(t)<1$ such that $0 \leq r-t<\delta(t)$ implies $\eta(r) \leq s(t)$.

This property obviously holds if $\eta$ is continuous since $\eta$ attains its maximum (less than 1 ) on each compact $[t, t+\delta(t)]$.

Definition 22 A sequence $\left\{x_{n}\right\}$ is said to be asymptotically $T$-regular with respect to $f$ if $\lim _{n \rightarrow \infty} d\left(f x_{n}, T x_{n}\right)=0$. 
The following theorem is related to the main results of $\mathrm{Hu}$ [25, Theorem 2], Jungck [14], Kaneko [26], Nadler [24, Theorem 5] and Beg and Azam [6, Theorem 5.4 and Corollary 5.5].

Theorem 23 Let $Y$ be an arbitrary non-empty set, $(X, d)$ be a metric space, $f: Y \rightarrow X$ and $T: Y \rightarrow C L(X)$ be such that condition (1) holds and

$$
\int_{0}^{H(T x, T y)} \psi(t) d t<\eta(d(f x, f y)) \int_{0}^{d(f x, f y)} \psi(t) d t
$$

for all $x, y \in Y$, where $\eta:[0, \infty) \rightarrow[0,1)$ satisfies $(\mathcal{P})$ and $\psi \geq 0$ is nonincreasing.

Suppose also that $T x$ is a compact set for every $x \in Y$.

If $(Y)$ is complete, then

(i) there exists an asymptotically $T$-regular sequence $\left\{x_{n}\right\}$ with respect to $f$ in $Y$,

(ii) $f$ and $T$ have a coincidence point.

Further, if $f$ and $T$ are occasionally coincidentally idempotent, then $f$ and $T$ have a common fixed point.

Proof For some $x_{0}$ in $Y$, let $y_{0}=f x_{0}$ and choose $x_{1}$ in $Y$ such that $y_{1}=f x_{1} \in T x_{0}$. Then, by (20), we have

$$
\int_{0}^{H\left(T x_{0}, T x_{1}\right)} \psi(t) d t<\eta\left(d\left(f x_{0}, f x_{1}\right)\right) \int_{0}^{d\left(f x_{0}, f x_{1}\right)} \psi(t) d t .
$$

Using (1), we can choose $x_{2} \in Y$ such that $y_{2}=f x_{2} \in T x_{1}$ and satisfying that

$$
d\left(y_{1}, y_{2}\right)=d\left(f x_{1}, y_{2}\right)=d\left(f x_{1}, T x_{1}\right) \leq H\left(T x_{0}, T x_{1}\right),
$$

hence

$$
\begin{aligned}
\int_{0}^{d\left(y_{1}, y_{2}\right)} \psi(t) d t & =\int_{0}^{d\left(f x_{1}, f x_{2}\right)} \psi(t) d t<\eta\left(d\left(f x_{0}, f x_{1}\right)\right) \int_{0}^{d f\left(f x_{0}, f x_{1}\right)} \psi(t) d t \\
& \leq \int_{0}^{d f\left(f x_{0}, f x_{1}\right)} \psi(t) d t .
\end{aligned}
$$

Note that, in the previous inequalities, we have used that $d\left(f x_{0}, f x_{1}\right)>0$. If $d\left(f x_{0}, f x_{1}\right)=0$, then $f x_{0}=f x_{1} \in T x_{0}$ and $\left\{x_{n}\right\}$ is asymptotically $T$-regular with respect to $f$.

By induction, we construct a sequence $\left\{x_{n}\right\}$ in $Y$ and $\left\{y_{n}\right\}$ in $f(Y)$ such that, for every $n$,

$$
d\left(f x_{n-1}, y_{n}\right)=d\left(f x_{n-1}, T x_{n-1}\right)=\min _{y \in T x_{n-1}} d\left(f x_{n-1}, y\right) \leq H\left(T x_{n-2}, T x_{n-1}\right),
$$

and $y_{n}=f x_{n} \in T x_{n-1}$.

Also, we have

$$
\begin{aligned}
& \int_{0}^{d\left(y_{n+1}, y_{n+2}\right)} \psi(t) d t \\
& \quad=\int_{0}^{d\left(f x_{n+1}, f x_{n+2}\right)} \psi(t) d t
\end{aligned}
$$




$$
\begin{aligned}
& =\int_{0}^{d\left(f x_{n+1}, T x_{n+1}\right)} \psi(t) d t \leq \int_{0}^{H\left(T x_{n}, T x_{n+1}\right)} \psi(t) d t \\
& \leq \eta\left(d\left(f x_{n}, f x_{n+1}\right)\right) \int_{0}^{\left.d f x_{n}, f x_{n+1}\right)} \psi(t) d t \\
& <\int_{0}^{d\left(f x_{n}, f x_{n+1}\right)} \psi(t) d t=\int_{0}^{d\left(y_{n}, y_{n+1}\right)} \psi(t) d t .
\end{aligned}
$$

It follows that the sequence $\left\{d\left(y_{n}, y_{n+1}\right)\right\}$ is decreasing and converges to its greatest lower bound, say $t$. Clearly $t \geq 0$. If $t>0$, then by the property $(\mathcal{P})$ of $\eta$, there will exist $\delta(t)>0$ and $s(t)<1$ such that

$$
0 \leq r-t<\delta(t) \quad \text { implies } \quad \eta(r) \leq s(t)
$$

For this $\delta(t)>0$, there exists $N \in \mathbb{N}$ such that $0 \leq d\left(y_{n}, y_{n+1}\right)-t<\delta(t)$, whenever $n \geq N$. Hence $\eta\left(d\left(y_{n}, y_{n+1}\right)\right) \leq s(t)$, whenever $n \geq N$. Let $K=\max \left\{\eta\left(d\left(y_{0}, y_{1}\right)\right), \eta\left(d\left(y_{1}, y_{2}\right)\right), \ldots\right.$, $\left.\eta\left(d\left(y_{N-1}, y_{N}\right)\right), s(t)\right\}$. Then for $n=1,2,3, \ldots$, we have

$$
\begin{aligned}
\int_{0}^{d\left(y_{n}, y_{n+1}\right)} \psi(t) d t & <\eta\left(d\left(y_{n-1}, y_{n}\right)\right) \int_{0}^{d\left(y_{n-1}, y_{n}\right)} \psi(t) d t \\
& \leq K \int_{0}^{d\left(y_{n-1}, y_{n}\right)} \psi(t) d t \\
& \leq K^{n} \int_{0}^{d\left(y_{0}, y_{1}\right)} \psi(t) d t \rightarrow 0 \text { as } n \rightarrow \infty,
\end{aligned}
$$

which contradicts the assumption that $t>0$. Thus $\lim _{n \rightarrow \infty} d\left(y_{n}, y_{n+1}\right)=0$; i.e., $d\left(f x_{n}\right.$, $\left.T x_{n}\right) \rightarrow 0$ as $n \rightarrow+\infty$. Hence the sequence $\left\{x_{n}\right\}$ is asymptotically $T$-regular with respect to $f$.

We claim that $\left\{f x_{n}\right\}$ is a Cauchy sequence. Let $n, m \in \mathbb{N}$ with $n<m$, then, by the nonincreasing character of $\psi$, we get

$$
\begin{aligned}
& \int_{0}^{d\left(y_{n}, y_{m}\right)} \psi(t) d t \\
& \quad \leq \int_{0}^{d\left(y_{n}, y_{n+1}\right)+d\left(y_{n+1}, y_{n+2}\right)+\cdots+d\left(y_{m-1}, y_{m}\right)} \psi(t) d t=\int_{0}^{d\left(y_{n}, y_{n+1}\right)} \psi(t) d t \\
& \quad+\int_{d\left(y_{n}, y_{n+1}\right)}^{d\left(y_{n}, y_{n+1}\right)+d\left(y_{n+1}, y_{n+2}\right)} \psi(t) d t+\cdots+\int_{d\left(y_{n}, y_{n+1}\right)+d\left(y_{n+1}, y_{n+2}\right)+\cdots+d\left(y_{m-2}, y_{m-1}\right)}^{d\left(y_{n}, y_{n+1}\right)+d\left(y_{n+1}, y_{n+2}\right)+\cdots+d\left(y_{m-1}, y_{m}\right)} \psi(t) d t \\
& \leq \int_{0}^{d\left(y_{n}, y_{n+1}\right)} \psi(t) d t+\int_{0}^{d\left(y_{n+1}, y_{n+2}\right)} \psi(t) d t+\cdots+\int_{0}^{d\left(y_{m-1}, y_{m}\right)} \psi(t) d t \\
& =\sum_{i=n}^{m-1} \int_{0}^{d\left(y_{i}, y_{i+1}\right)} \psi(t) d t .
\end{aligned}
$$

Now, we recall that

$$
\int_{0}^{d\left(y_{n+1}, y_{n+2}\right)} \psi(t) d t \leq \eta\left(d\left(y_{n}, y_{n+1}\right)\right) \int_{0}^{d\left(y_{n}, y_{n+1}\right)} \psi(t) d t
$$


for every $n$, which implies that

$$
\begin{aligned}
\int_{0}^{d\left(y_{n+2}, y_{n+3}\right)} \psi(t) d t & \leq \eta\left(d\left(y_{n+1}, y_{n+2}\right)\right) \int_{0}^{d\left(y_{n+1}, y_{n+2}\right)} \psi(t) d t \\
& \leq \eta\left(d\left(y_{n+1}, y_{n+2}\right)\right) \eta\left(d\left(y_{n}, y_{n+1}\right)\right) \int_{0}^{d\left(y_{n}, y_{n+1}\right)} \psi(t) d t .
\end{aligned}
$$

Following this procedure, we prove that

$$
\int_{0}^{d\left(y_{j}, y_{j+1}\right)} \psi(t) d t \leq \prod_{i=n}^{j-1} \eta\left(d\left(y_{i}, y_{i+1}\right)\right) \int_{0}^{d\left(y_{n}, y_{n+1}\right)} \psi(t) d t, \quad \text { for every } j=n+1, \ldots, m-1 .
$$

Therefore,

$$
\begin{aligned}
\int_{0}^{d\left(y_{n}, y_{m}\right)} \psi(t) d t & \leq \sum_{i=n}^{m-1} \int_{0}^{d\left(y_{i}, y_{i+1}\right)} \psi(t) d t \\
& =\int_{0}^{d\left(y_{n}, y_{n+1}\right)} \psi(t) d t+\sum_{i=n+1}^{m-1} \int_{0}^{d\left(y_{i}, y_{i+1}\right)} \psi(t) d t \\
& \leq\left[1+\sum_{i=n+1}^{m-1} \prod_{l=n}^{i-1} \eta\left(d\left(y_{l}, y_{l+1}\right)\right)\right] \int_{0}^{d\left(y_{n}, y_{n+1}\right)} \psi(t) d t .
\end{aligned}
$$

We check that the right-hand side in the last inequality tends to 0 as $n, m \rightarrow+\infty$. Since $\int_{0}^{d\left(y_{n}, y_{n+1}\right)} \psi(t) d t \rightarrow 0$ as $n \rightarrow+\infty$, it suffices to show that $\sum_{i=n+1}^{m-1} \prod_{l=n}^{i-1} \eta\left(d\left(y_{l}, y_{l+1}\right)\right)$ is bounded (uniformly on $n, m$ ). Indeed, we check that $\sum_{i=n+1}^{m-1} \prod_{l=n}^{i-1} \eta\left(z_{l}\right)$ is bounded for any sequence $\left\{z_{l}\right\}$ with nonnegative terms and tending to 0 as $l \rightarrow+\infty$, using the property $(\mathcal{P})$ of the function $\eta$. Given $t=0$, by $(\mathcal{P})$, there exist $\delta(0)>0, s_{0}<1$ such that $0 \leq r<\delta(0)$ implies $\eta(r) \leq s_{0}$. Since $\left\{z_{l}\right\} \rightarrow 0$, given $\delta(0)>0$, there exists $l_{0} \in \mathbb{N}$ such that, for every $l \geq l_{0}$, we have $0 \leq z_{l}<\delta(0)$. This implies that $\eta\left(z_{l}\right) \leq s_{0}$ for every $l \geq l_{0}$.

In consequence, for $n \geq l_{0}$, we get

$$
\begin{aligned}
0 & \leq \sum_{i=n+1}^{m-1} \prod_{l=n}^{i-1} \eta\left(z_{l}\right) \leq \sum_{i=n+1}^{m-1} \prod_{l=n}^{i-1} s_{0}=\sum_{i=n+1}^{m-1}\left(s_{0}\right)^{i-n} \\
& =\frac{s_{0}-\left(s_{0}\right)^{m-n}}{1-s_{0}}<\frac{s_{0}}{1-s_{0}}
\end{aligned}
$$

and this expression is bounded independently of $m, n$.

Hence $\left\{f x_{n}\right\}$ is a Cauchy sequence in $f(Y)$. Since $f(Y)$ is complete, $\left\{x_{n}\right\}$ converges to some $p$ in $f(Y)$. Let $z \in f^{-1}(p)$. Then $f z=p$. Next, we have

$$
\begin{aligned}
\int_{0}^{d(f z, T z)} \psi(t) d t & \leq \int_{0}^{d\left(f x_{n+1}, f z\right)+d\left(f x_{n+1}, T z\right)} \psi(t) d t \\
& =\int_{0}^{d\left(f x_{n+1}, T z\right)} \psi(t) d t+\int_{d\left(f x_{n+1}, T z\right)}^{d\left(f x_{n+1}, T z\right)+d\left(f z, f x_{n+1}\right)} \psi(t) d t \\
& \leq \int_{0}^{d\left(f x_{n+1}, T z\right)} \psi(t) d t+\int_{0}^{d\left(f z, x_{n+1}\right)} \psi(t) d t
\end{aligned}
$$




$$
\begin{aligned}
& \leq \int_{0}^{H\left(T x_{n}, T z\right)} \psi(t) d t+\int_{0}^{d\left(f z, f x_{n+1}\right)} \psi(t) d t \\
& \leq \eta\left(d\left(f x_{n}, f z\right)\right) \int_{0}^{d\left(f x_{n}, f z\right)} \psi(t) d t+\int_{0}^{d\left(f z, f x_{n+1}\right)} \psi(t) d t .
\end{aligned}
$$

Letting $n \rightarrow \infty$, we get $\int_{0}^{d(f z, T z)} \psi(t) d t \leq 0$. Thus we have $d(f z, T z)=0$. Hence $f z \in T z$.

Now, if $f$ and $T$ are occasionally coincidentally idempotent, then $f f w=f w$ for some $w \in$ $C(T, f)$. Then we have

$$
\int_{0}^{H(T f w, T w)} \psi(t) d t \leq \eta(d(f f w, f w)) \int_{0}^{d(f f w, f w)} \psi(t) d t=0 .
$$

Thus, $T f w=T w$. It follows that $f f w=f w \in T w=T f w$. Hence, $f w$ is a common fixed point of $T$ and $f$.

Now we state some fixed point theorems for Kannan-type multi-valued mappings which extend and generalize the corresponding results of Shiau et al. [10] and Beg and Azam $[6,27]$. A proper blend of the proof of Theorem 9 and those of $[10$, Th. 6, Th. 7, Th. 8 respectively] and [9, Theorems 3.1,3.2, 3.3] will complete the proof.

Theorem 24 Let $Y$ be an arbitrary non-empty set, $(X, d)$ be a metric space, $f: Y \rightarrow X$ and $T: Y \rightarrow C B(X)$ be such that (1) holds and

$$
\begin{aligned}
& \int_{0}^{H^{r}(T x, T y)} \psi(t) d t \\
& \quad \leq \alpha_{1}(d(f x, T x)) \int_{0}^{d^{r}(f x, T x)} \psi(t) d t+\alpha_{2}(d(f y, T y)) \int_{0}^{d^{r}(f y, T y)} \psi(t) d t
\end{aligned}
$$

for all $x, y \in Y$, where $\alpha_{i}: \mathbb{R}_{+} \rightarrow[0,1)(i=1,2)$ are bounded on bounded sets, $r$ is some fixed positive real number and $\psi: \mathbb{R}_{+} \rightarrow \mathbb{R}_{+}$is a Lebesgue measurable mapping which is summable on each compact interval and $\int_{0}^{\epsilon} \psi(t) d t>0$ for each $\epsilon>0$. Suppose that there exists an asymptotically $T$-regular sequence $\left\{x_{n}\right\}$ with respect to $f$ in $Y$.If $T(Y)$ is complete or

there exists $k \in \mathbb{N}$ such that $f x_{n+k} \in T x_{n} \quad$ for every $n \in \mathbb{N}$, and $f(Y)$ is complete,

then $f$ and $T$ have a coincidence point. Further, iff and $T$ are occasionally coincidentally idempotent, then $f$ and $T$ have a common fixed point.

Proof By hypotheses,

$$
\begin{aligned}
& \int_{0}^{H^{r}\left(T x_{n}, T x_{m}\right)} \psi(t) d t \\
& \quad \leq \alpha_{1}\left(d\left(f x_{n}, T x_{n}\right)\right) \int_{0}^{d^{r}\left(f x_{n}, T x_{n}\right)} \psi(t) d t+\alpha_{2}\left(d\left(f x_{m}, T x_{m}\right)\right) \int_{0}^{d^{r}\left(f x_{m}, T x_{m}\right)} \psi(t) d t .
\end{aligned}
$$

Since $\left\{x_{n}\right\}$ is asymptotically $T$-regular with respect to $f$ in $Y$, then $\left\{\alpha_{1}\left(d\left(f x_{n}, T x_{n}\right)\right)\right\}_{n}$ and $\left\{\alpha_{2}\left(d\left(f x_{m}, T x_{m}\right)\right)\right\}_{m}$ are bounded sequences and $\int_{0}^{d^{r}\left(f x_{n}, T x_{n}\right)} \psi(t) d t \rightarrow 0$, 
$\int_{0}^{d^{r}\left(f x_{m}, T x_{m}\right)} \psi(t) d t \rightarrow 0$, as $n, m \rightarrow+\infty$. This provides the property $H\left(T x_{n}, T x_{m}\right) \rightarrow 0$ as $n, m \rightarrow+\infty$, so that $\left\{T x_{n}\right\}$ is a Cauchy sequence in $(C B(X), H)$.

If $T(Y)$ is complete, there exists $K^{*} \in T(Y) \subseteq f(Y)$ such that $H\left(T x_{n}, K^{*}\right) \rightarrow 0$ as $n \rightarrow+\infty$. Let $u \in Y$ be such that $f(u) \in K^{*}$. Then

$$
\begin{aligned}
\int_{0}^{d^{r}(f u, T u)} \psi(t) d t \leq & \int_{0}^{H^{r}\left(K^{*}, T u\right)} \psi(t) d t \leq \int_{0}^{\left(H\left(K^{*}, T x_{n}\right)+H\left(T x_{n}, T u\right)\right)^{r}} \psi(t) d t \\
& =\int_{0}^{H^{r}\left(T x_{n}, T u\right)} \psi(t) d t+\int_{H^{r}\left(T x_{n}, T u\right)}^{H^{r}\left(T x_{n}, T u\right)+\text { terms containing } H\left(K^{*}, T x_{n}\right)} \psi(t) d t \\
\leq & \alpha_{1}\left(d\left(f x_{n}, T x_{n}\right)\right) \int_{0}^{d^{r}\left(f x_{n}, T x_{n}\right)} \psi(t) d t+\alpha_{2}(d(f u, T u)) \int_{0}^{d^{r}(f u, T u)} \psi(t) d t \\
& +\int_{H^{r}\left(T x_{n}, T u\right)}^{H^{r}\left(T x_{n}, T u\right)+\text { terms containing } H\left(K^{*}, T x_{n}\right)} \psi(t) d t,
\end{aligned}
$$

where the number of terms containing $H\left(K^{*}, T x_{n}\right)$ is a finite number depending on $r$, and therefore fixed. Calculating the limit as $n \rightarrow+\infty$ and taking into account that the length of the intervals in the last integral tends to zero, we get

$$
\int_{0}^{d^{r}(f u, T u)} \psi(t) d t\left(1-\alpha_{2}(d(f u, T u))\right) \leq \lim _{n \rightarrow+\infty} \alpha_{1}\left(d\left(f x_{n}, T x_{n}\right)\right) \int_{0}^{d^{r}\left(f x_{n}, T x_{n}\right)} \psi(t) d t=0 .
$$

Therefore,

$$
\int_{0}^{d^{r}(f u, T u)} \psi(t) d t \leq 0
$$

and, by the properties of $\psi$, we get $d^{r}(f u, T u)=0$, which implies that $f u \in T u$ and $u$ is a coincidence point.

Now, suppose that $f(Y)$ is complete. Note that $T x_{n}$ is closed and bounded for every $n \in \mathbb{N}$. Take $k>1$ fixed. By the results in [24], we can affirm that for every $y_{1} \in T x_{n}$, there exists $y_{2} \in T x_{m}$ such that $d\left(y_{1}, y_{2}\right) \leq k H\left(T x_{n}, T x_{m}\right)$.

Given $n, m \in \mathbb{N}$, we choose $y_{1} \in T x_{n}$ and, for this $y_{1} \in T x_{n}$ fixed, we choose $y_{2} \in T x_{m}$ such that $d\left(y_{1}, y_{2}\right) \leq k H\left(T x_{n}, T x_{m}\right)$. Then

$$
\begin{aligned}
d\left(f x_{n}, f x_{m}\right) & \leq d\left(f x_{n}, y_{1}\right)+d\left(y_{1}, y_{2}\right)+d\left(y_{2}, f x_{m}\right) \\
& \leq d\left(f x_{n}, T x_{n}\right)+k H\left(T x_{n}, T x_{m}\right)+d\left(T x_{m}, f x_{m}\right) .
\end{aligned}
$$

By the hypothesis on $\left\{x_{n}\right\}$ and the Cauchy character of $\left\{T x_{n}\right\}$, we deduce that $\left\{f x_{n}\right\}$ is a Cauchy sequence. Since $f(Y)$ is complete, there exists $f(u) \in f(Y)$ such that $\left\{f\left(x_{n}\right)\right\} \rightarrow f(u)$. By hypotheses, $d\left(f x_{n+k}, T u\right) \leq H\left(T x_{n}, T u\right)$ for every $n$, hence

$$
\begin{aligned}
& \int_{0}^{d^{r}\left(f x_{n+k}, T u\right)} \psi(t) d t \\
& \quad \leq \int_{0}^{H^{r}\left(T x_{n}, T u\right)} \psi(t) d t \\
& \quad \leq \alpha_{1}\left(d\left(f x_{n}, T x_{n}\right)\right) \int_{0}^{d^{r}\left(f x_{n}, T x_{n}\right)} \psi(t) d t+\alpha_{2}(d(f u, T u)) \int_{0}^{d^{r}(f u, T u)} \psi(t) d t,
\end{aligned}
$$


and taking the limit as $n \rightarrow+\infty$, we get

$$
\int_{0}^{d^{r}(f u, T u)} \psi(t) d t \leq \alpha_{2}(d(f u, T u)) \int_{0}^{d^{r}(f u, T u)} \psi(t) d t .
$$

In this case,

$$
\left(1-\alpha_{2}(d(f u, T u))\right) \int_{0}^{d^{r}(f u, T u)} \psi(t) d t \leq 0
$$

and $d(f u, T u)=0$, which implies that $f u \in T u$. Now, if $f$ and $T$ are coincidentally idempotent, then $f f w=f w$ for some $w \in C(T, f)$. Hence

$$
\begin{aligned}
& \int_{0}^{H^{r}(T f w, T w)} \psi(t) d t \\
& \quad \leq \alpha_{1}(d(f f w, T f w)) \int_{0}^{d^{r}(f f w, T f w)} \psi(t) d t+\alpha_{2}(d(f w, T w)) \int_{0}^{d^{r}(f w, T w)} \psi(t) d t \\
& \quad=\alpha_{1}(d(f w, T f w)) \int_{0}^{d^{r}(f w, T f w)} \psi(t) d t .
\end{aligned}
$$

Since $f f w=f w \in T w$, we get

$$
\int_{0}^{d^{r}(f w, T f w)} \psi(t) d t \leq \int_{0}^{H^{r}(T w, T f w)} \psi(t) d t \leq \alpha_{1}(d(f w, T f w)) \int_{0}^{d^{r}(f w, T f w)} \psi(t) d t .
$$

Therefore

$$
\int_{0}^{d^{r}(f w, T f w)} \psi(t) d t\left(1-\alpha_{1}(d(f w, T f w))\right) \leq 0,
$$

obtaining $d(f w, T f w)=0$ and $f w \in T f w$. Since $0 \leq \int_{0}^{H^{r}(T f w, T w)} \psi(t) d t \leq 0$, we deduce that $H(T f w, T w)=0$ and $T f w=T w$. In consequence, $f f w=f w \in T w=T f w$ and $f w$ is a common fixed point of $T$ and $f$.

Remark 25 In the statement of Theorem 24, condition (22) can be replaced by the more general one

$$
f(Y) \text { is complete. }
$$

To complete the proof with this more general hypothesis, take into account that for $y \in Y$, $T(y)$ is a closed set in $X$ and $T(Y) \subseteq f(Y)$. Using that $f(Y)$ is complete, we deduce that $(C L(f(Y)), H)$ is complete. Hence $\left\{T_{n}\right\}$ is a sequence in $C L(f(Y))$ and it is a Cauchy sequence in $(C L(f(Y)), H)$. Therefore, there exists $K^{*} \in C L(f(Y))$ such that $H\left(T x_{n}, K^{*}\right) \rightarrow 0$ as $n \rightarrow+\infty$. Note also that $K^{\prime \prime}$ is a closed set in the complete space $f(Y)$, then $K^{\prime \prime}$ is complete and, therefore, a closed set, then $K^{*} \in C L(X)$. Once we have proved that $H\left(T x_{n}, K^{*}\right) \rightarrow 0$ as $n \rightarrow+\infty$ in $(C L(f(Y)), H)$, the proof follows analogously.

Theorem 26 In addition to the hypotheses of Theorem 24, suppose that $T x_{n}$ is compact for all $n \in \mathbb{N}$. If $f(z)$ is a cluster point of $\left\{x_{n}\right\}$, then $z$ is a coincidence point off and $T$. 
Proof Let $y_{n} \in T x_{n}$ be such that $d\left(f x_{n}, y_{n}\right)=d\left(f x_{n}, T x_{n}\right) \rightarrow 0$, this is possible since $T x_{n}$ is compact. It is obvious that a cluster point of $\left\{x_{n}\right\}$ is a cluster point of $\left\{y_{n}\right\}$. Let $f(z)$ be a cluster point of $\left\{x_{n}\right\}$ and $\left\{y_{n}\right\}$, then we check that $f z \in T u$, where $u$ is obtained in the proof of Theorem 24. Note that, for every $y \in T u$,

$$
d(f z, y) \leq d\left(f z, f x_{n}\right)+d\left(f x_{n}, y_{n}\right)+d\left(y_{n}, y\right)=d\left(f z, f x_{n}\right)+d\left(f x_{n}, T x_{n}\right)+d\left(y_{n}, y\right),
$$

hence

$$
\begin{aligned}
d(f z, T u) & =\inf _{y \in T u} d(f z, y) \leq d\left(f z, f x_{n}\right)+d\left(f x_{n}, T x_{n}\right)+\inf _{y \in T u} d\left(y_{n}, y\right) \\
& =d\left(f z, f x_{n}\right)+d\left(f x_{n}, T x_{n}\right)+d\left(y_{n}, T u\right) \leq d\left(f z, f x_{n}\right)+d\left(f x_{n}, T x_{n}\right)+H\left(T x_{n}, T u\right) .
\end{aligned}
$$

In consequence,

$$
\int_{0}^{d^{r}(f z, T u)} \psi(t) d t \leq \int_{0}^{\left.\left(d f f z, f x_{n}\right)+d\left(f x_{n}, T x_{n}\right)+H\left(T x_{n}, T u\right)\right)^{r}} \psi(t) d t .
$$

Using that there exists a subsequence $f x_{n_{k}}$ converging to $f z$, the properties of $\left\{x_{n}\right\}$ and the inequality

$$
\begin{aligned}
& \int_{0}^{H^{r}\left(T x_{n_{k}}, T u\right)} \psi(t) d t \\
& \quad \leq \alpha_{1}\left(d\left(f x_{n_{k}}, T x_{n_{k}}\right)\right) \int_{0}^{d^{r}\left(f x_{n_{k}}, T x_{n_{k}}\right)} \psi(t) d t+\alpha_{2}(d(f u, T u)) \int_{0}^{d^{r}(f u, T u)} \psi(t) d t \stackrel{k \rightarrow+\infty}{\longrightarrow} 0,
\end{aligned}
$$

then, taking the limit when $n_{k} \rightarrow+\infty$, we get $\int_{0}^{d^{r}(f z, T u)} \psi(t) d t \leq 0$ and $f z \in T u$. To prove that $f z \in T z$, using that $f u \in T u$, we get

$$
\begin{aligned}
\int_{0}^{d^{r}(f z, T z)} \psi(t) d t & \leq \int_{0}^{H^{r}(T u, T z)} \psi(t) d t \\
& \leq \alpha_{1}(d(f u, T u)) \int_{0}^{d^{r}(f u, T u)} \psi(t) d t+\alpha_{2}(d(f z, T z)) \int_{0}^{d^{r}(f z, T z)} \psi(t) d t \\
& =\alpha_{2}(d(f z, T z)) \int_{0}^{d^{r}(f z, T z)} \psi(t) d t .
\end{aligned}
$$

This implies that

$$
\left(1-\alpha_{2}(d(f z, T z))\right) \int_{0}^{d^{r}(f z, T z)} \psi(t) d t \leq 0
$$

and, by the properties of $\alpha_{2}$ and $\psi$, we deduce that $d(f z, T z)=0$, which proves that $z$ is a coincidence point of $f$ and $T$.

The following result extends [10, Theorem 3.3].

Theorem 27 Let $Y$ be an arbitrary non-empty set, $(X, d)$ be a metric space, $f: Y \rightarrow X$ and $T: Y \rightarrow C B(X)$ be such that $(1)$ and $(21)$ hold, where $\alpha_{i}: \mathbb{R}_{+} \rightarrow[0,1)(i=1,2)$ are bounded 
on bounded sets and such that

$$
\alpha_{1}(d(f x, T x))+\alpha_{2}(d(f y, T y)) \leq 1 \quad \text { for every } x, y,
$$

$r$ is some fixed positive real number and $\psi: \mathbb{R}_{+} \rightarrow \mathbb{R}_{+}$is a Lebesgue measurable mapping which is summable on each compact interval, and $\psi(x)>0$ for each $x>0$. Suppose that

$$
\inf \left\{d\left(f z_{n}, T z_{n}\right): n \in \mathbb{N}\right\}=0 \quad \text { for every sequence }\left\{z_{n}\right\} \text { in } Y \text { with } f z_{n} \in T z_{n-1}, \forall n \text {. }
$$

If $T(Y)$ is complete or $f(Y)$ is complete, then $f$ and $T$ have a coincidence point. Further, if $f$ and $T$ are occasionally coincidentally idempotent, then $f$ and $T$ have a common fixed point.

Proof Using Theorem 24, it suffices to prove that there exists an asymptotically $T$-regular sequence $\left\{x_{n}\right\}$ with respect to $f$ in $Y$. Let $x_{0} \in Y$ and take $\left\{x_{n}\right\}$ in $Y$ such that $f x_{n} \in T x_{n-1}$ for every $n \in \mathbb{N}$. Then

$$
\begin{aligned}
& \int_{0}^{d^{r}\left(f x_{n}, T x_{n}\right)} \psi(t) d t \\
& \quad \leq \int_{0}^{H^{r}\left(T x_{n-1}, T x_{n}\right)} \psi(t) d t \\
& \quad \leq \alpha_{1}\left(d\left(f x_{n-1}, T x_{n-1}\right)\right) \int_{0}^{d^{r}\left(f x_{n-1}, T x_{n-1}\right)} \psi(t) d t+\alpha_{2}\left(d\left(f x_{n}, T x_{n}\right)\right) \int_{0}^{d^{r}\left(f x_{n}, T x_{n}\right)} \psi(t) d t .
\end{aligned}
$$

Hence,

$$
\left(1-\alpha_{2}\left(d\left(f x_{n}, T x_{n}\right)\right)\right) \int_{0}^{d^{r}\left(f x_{n}, T x_{n}\right)} \psi(t) d t \leq \alpha_{1}\left(d\left(f x_{n-1}, T x_{n-1}\right)\right) \int_{0}^{d^{r}\left(f x_{n-1}, T x_{n-1}\right)} \psi(t) d t,
$$

or also, using the hypothesis on $\alpha_{1}$ and $\alpha_{2}$,

$$
\begin{aligned}
\int_{0}^{d^{r}\left(f x_{n}, T x_{n}\right)} \psi(t) d t & \leq \frac{\alpha_{1}\left(d\left(f x_{n-1}, T x_{n-1}\right)\right)}{\left(1-\alpha_{2}\left(d\left(f x_{n}, T x_{n}\right)\right)\right)} \int_{0}^{d^{r}\left(f x_{n-1}, T x_{n-1}\right)} \psi(t) d t \\
& \leq \int_{0}^{d^{r}\left(f x_{n-1}, T x_{n-1}\right)} \psi(t) d t .
\end{aligned}
$$

The properties of $\psi$ imply that $d^{r}\left(f x_{n}, T x_{n}\right) \leq d^{r}\left(f x_{n-1}, T x_{n-1}\right)$ for every $n \in \mathbb{N}$, and $\left\{d\left(f x_{n}, T x_{n}\right)\right\}_{n \in \mathbb{N}}$ is nonincreasing and bounded below. Therefore it is convergent to the infimum, that is,

$$
d\left(f x_{n}, T x_{n}\right) \rightarrow \inf \left\{d\left(f x_{n}, T x_{n}\right): n \in \mathbb{N}\right\}=0,
$$

and $\left\{x_{n}\right\}$ is asymptotically $T$-regular with respect to $f$ in $Y$.

Remark 28 Note that condition (23) in Theorem 27 cannot be replaced by

$$
\inf \{d(f x, T x): x \in Y\}=0
$$


since the infimum taking the sequence $\left\{z_{n}\right\}$ could be positive (we calculate the infimum in a smaller set).

Remark 29 In Theorem 27, condition (23) can be replaced by the following:

$$
\inf \left\{H\left(T z_{n-1}, T z_{n}\right): n \in \mathbb{N}\right\}=0,
$$

for every sequence $\left\{z_{n}\right\}$ in $Y$ with $f z_{n} \in T z_{n-1}, \forall n$.

Indeed, since

$$
d\left(f z_{n}, T z_{n}\right) \leq H\left(T z_{n-1}, T z_{n}\right), \quad \forall n
$$

then

$$
0 \leq \inf \left\{d\left(f z_{n}, T z_{n}\right): n \in \mathbb{N}\right\} \leq \inf \left\{H\left(T z_{n-1}, T z_{n}\right): n \in \mathbb{N}\right\}=0,
$$

and $d\left(f z_{n}, T z_{n}\right) \rightarrow 0$.

Remark 30 In Theorem 27, if we are able to obtain a sequence $\left\{x_{n}\right\}$ with an infinite number of terms which are different, then we can relax condition (23) to the following:

$$
\inf \{d(f x, T x): x \in B\}=0 \quad \text { for every infinite set } B \text { of } Y \text {. }
$$

Competing interests

The authors declare that they have no competing interests.

Authors' contributions

Both authors contributed to each part of this work equally and read and approved the final version of the manuscript.

\section{Author details}

${ }^{1}$ School of Studies in Mathematics, Pt. Ravishankar Shukla University, Raipur, C.G. 492010, India. ${ }^{2}$ Departamento de Análisis Matemático, Facultad de Matemáticas, Universidad de Santiago de Compostela, Santiago de Compostela, 15782, Spain.

\section{Acknowledgements}

Dedicated to Professor Jean Mawhin, on the occasion of his seventieth birthday.

We thank the editor, the anonymous referees and also Professor Stojan Radenović for their helpful comments and suggestions. This research was partially supported by the University Grants Commission, New Delhi, India; Ministerio de Economía y Competitividad, project MTM2010-15314, and co-financed by EC fund FEDER.

Received: 10 December 2012 Accepted: 25 May 2013 Published: 11 June 2013

\section{References}

1. Shiau, C, Tan, KK, Wong, CS: A class of quasi-nonexpansive multi-valued maps. Can. Math. Bull. 18, $707-714$ (1975)

2. Rus, IA: Generalized Contractions and Applications. Cluj University Press, Cluj-Napoca (2001)

3. Kikkawa, M, Suzuki, T: Three fixed point theorems for generalized contractions with constants in complete metric spaces. Nonlinear Anal., Theory Methods Appl. 69(9), 2942-2949 (2008)

4. Dhompongsa, S, Yingtaweesittikul, H: Fixed points for multivalued mappings and the metric completeness. Fixed Point Theory Appl. 2009, Article ID 972395 (2009)

5. Moţ, G, Petruşel, A: Fixed point theory for a new type of contractive multivalued operators. Nonlinear Anal., Theory Methods Appl. 70(9), 3371-3377 (2009)

6. Beg, I, Azam, A: Fixed points of asymptotically regular multivalued mappings. J. Aust. Math. Soc. A 53, 313-326 (1992)

7. Naimpally, S, Singh, S, Whitefield, JHM: Coincidence theorems for hybrid contractions. Math. Nachr. 127, 177-180 (1986)

8. Pathak, HK: Fixed point theorems for weak compatible multi-valued and single valued mappings. Acta Math. Hung. 67(1-2), 69-78 (1995) 
9. Pathak, HK, Kang, SM, Cho, YJ: Coincidence and fixed point theorems for nonlinear hybrid generalized contractions. Czechoslov. Math. J. 48(123), 341-357 (1998)

10. Pathak, HK, Khan, MS: Fixed and coincidence points of hybrid mappings. Arch. Math. 38, 201-208 (2002)

11. Singh, SL, Mishra, SN: Coincidences and fixed points of nonself hybrid contractions. J. Math. Anal. Appl. 256(2), 486-497 (2001)

12. Singh, SL, Mishra, SN: Coincidence theorems for certain classes of hybrid contractions. Fixed Point Theory Appl. 2010 Article ID 898109 (2010)

13. Jungck, G: Commuting mappings and fixed points. Am. Math. Mon. 83, 261-263 (1976)

14. Kaneko, H: Single-valued and multi-valued f-contractions. Boll. Unione Mat. Ital. 4-A, $29-33$ (1985)

15. Kaneko, H: A common fixed point of weakly commuting multi-valued mappings. Math. Jpn. 33(5), 741-744 (1988)

16. Singh, SL, Ha, KS, Cho, YJ: Coincidence and fixed points of nonlinear hybrid contractions. Int. J. Math. Math. Sci. 12(2), 247-256 (1989)

17. Jungck, G, Rhoades, BE: Fixed points theorems for occasionally weakly compatible mappings. Fixed Point Theory 7(2), 287-296 (2006)

18. Jungck, G, Rhoades, BE: Fixed points theorems for occasionally weakly compatible mappings, Erratum. Fixed Point Theory 9, 286-296 (2008)

19. Vetro, C: On Branciari's theorem for weakly compatible mappings. Appl. Math. Lett. 23, 700-705 (2010)

20. Vijayaraju, P, Rhoades, BE, Mohanraj, R: A fixed point theorem for a pair of maps satisfying a general contractive condition of integral type. Int. J. Math. Math. Sci. 15, 2359-2364 (2005)

21. Abbas, M, Rhoades, BE: Common fixed point theorems for hybrid pairs of occasionally weakly compatible mappings satisfying generalized contractive condition of integral type. Fixed Point Theory Appl. 2007, Article ID 54101 (2007)

22. Abbas, M, Khan, AR: Common fixed points of generalized contractive hybrid pairs in symmetric spaces. Fixed Point Theory Appl. 2009, Article ID 869407 (2009)

23. Kadelburg, Z, Radenović, S, Rakočević, V: Topological vector spaces valued cone metric spaces and fixed point theorems. Fixed Point Theory Appl. 2010, 170253 (2010)

24. Nadler, S: Multi-valued contraction mappings. Pac. J. Math. 20, 475-488 (1969)

25. Hu, T: Fixed point theorems for multivalued mappings. Can. Math. Bull. 23, 193-197 (1980)

26. Kubiak, T: Fixed point theorems for contractive type multi-valued mappings. Math. Jpn. 30, 89-101 (1985)

27. Beg, I, Azam, A: Fixed point theorems for Kannan mappings. Indian J. Pure Appl. Math. 17(11), 1270-1275 (1986)

doi:10.1186/1687-2770-2013-145

Cite this article as: Pathak and Rodríguez-López: Noncommutativity of mappings in hybrid fixed point results. Boundary Value Problems 2013 2013:145.

\section{Submit your manuscript to a SpringerOpen ${ }^{\ominus}$ journal and benefit from:}

- Convenient online submission

- Rigorous peer review

- Immediate publication on acceptance

- Open access: articles freely available online

- High visibility within the field

- Retaining the copyright to your article 